\title{
A CLASS OF WAVES IN A DEFORMED VISCOELASTIC SOLID*
}

\author{
BY M. A. HAYES (University of East Anglia, Norwich) \\ AND \\ R. S. RIVLIN (Lehigh University)
}

\begin{abstract}
A small-amplitude plane sinusoidal wave is propagated in an isotropic nonlinear viscoelastic material subjected to a static pure homogeneous deformation. The wave is polarized along one of the principal directions for the pure homogeneous deformation. The normals to the planes of constant phase and amplitude for the wave are perpendicular to each other and lie in the principal plane normal to the direction of polarization. It is found that the complex slowness for such a wave is independent of the orientation of the direction of propagation in the principal plane. The three complex slownesses corresponding to a particular class of waves of this type polarized along the three principal directions satisfy a relation which is independent of the detailed form of the constitutive equation.
\end{abstract}

1. Introduction. In a previous paper [1] the propagation of a plane sinusoidal wave of small amplitude in an isotropic viscoelastic solid subjected to an initial pure homogeneous deformation was discussed. The secular equation was obtained for a plane wave, for which the normals to the planes of constant phase and planes of constant amplitude are not necessarily parallel and are arbitrarily oriented to the principal directions for the pure homogeneous deformation. It was assumed that the Rivlin-Ericksen constitutive equation for an isotropic viscoelastic solid was applicable and that the material was such that when it was held at constant deformation, although stress relaxation may initially take place, a state of constant stress was eventually reached. It can be shown that for the deformations considered the constitutive equation for an isotropic solid with memory may be reduced to the Rivlin-Ericksen constitutive equation, subject only to limitations which are of a physically insignificant nature.

In the present paper, these results are applied to the case when the wave is linearly polarized in one of the principal directions for the pure homogeneous deformation and a particular case is considered when the planes of constant phase and amplitude are orthogonal, the normals to these planes lying in the plane normal to the direction of polarization. For the wave considered, the component of the complex slowness along the normal to the planes of constant amplitude (which is imaginary) is $i(=\sqrt{ }-1)$ times its component (which is real) along the normal to the planes of constant phase. Relations for the slownesses of such waves which are independent of the precise form of the constitutive equation are obtained. In the particular case when there is no initial pure homogeneous deformation, we are concerned with the propagation of sinusoidal waves in a linear isotropic viscoelastic solid. This has been discussed by Lockett [2].

* Received October 13, 1971. The research described in this paper was supported by a grant from the National Science Foundation to Lehigh University. 
In another paper [3] an expression for the mean energy flux vector, the direction of which is the ray direction for the wave, was obtained. This is applied to waves of the type considered in the present paper and it is shown that the ray direction is, in general, parallel neither to the normal to the planes of constant phase, nor to the normal to the planes of constant amplitude.

2. Basic equations. We consider the propagation of a plane sinusoidal wave in an isotropic viscoelastic solid subjected to an initial pure homogeneous deformation with extension ratios $\lambda_{1}, \lambda_{2}, \lambda_{3}$ and principal directions parallel to the axes of a rectangular cartesian coordinate system $x$. It is assumed that the material is such that when it is held at constant deformation, the stress may relax initially, but eventually attains a constant value which depends only on the constant state of deformation at which the material is held.

In the pure homogeneous deformation, a particle which is initially at $\xi_{\alpha}$ in the system $x$ moves to $X_{A}$, where

$$
X_{1}=\lambda_{1} \xi_{1}, \quad X_{2}=\lambda_{2} \xi_{2}, \quad X_{3}=\lambda_{3} \xi_{3} .
$$

Suppose that the wave is polarized in the direction of the $x_{3}$-axis and that the normals to the planes of constant phase and constant amplitude for the wave, which are not necessarily the same, lie in the $x_{1} x_{2}$-planes. Suppose, also, that as a result of the wave, the particle which was initially at $\xi_{\alpha}$ moves to $x_{i}$ in the system $x$. We write

$$
x_{i}=X_{i}+\epsilon u_{i},
$$

where, using the usual complex notation,

$$
u_{i}=U \delta_{i 3} \exp \left[i \omega\left(S_{1} X_{1}+S_{2} X_{2}-t\right)\right] \text {. }
$$

$U$ may, without loss of generality, be taken to be real and then $\epsilon U$ is the amplitude of the wave, $t$ denotes time, $\omega$ is the angular frequency of the wave and $\left(S_{1}, S_{2}, 0\right)$ is the complex slowness vector for the wave.

The Rivlin-Ericksen constitutive equation for an isotropic viscoelastic solid is assumed to be valid. Using this constitutive equation, it has been seen in a previous paper [1] that for the wave considered the components of the slowness vector are related by the equation

$$
\Psi_{31} S_{1}^{2}+\Psi_{32} S_{2}^{2}=\rho
$$

where $\rho$ is the density of the material in the state of pure homogeneous deformation considered and $\Psi_{\beta \gamma}$ are constitutive functions for the material which depend on $\lambda_{1}^{2}, \lambda_{2}^{2}, \lambda_{3}^{2}$ and $i \omega$ and are given by ${ }^{1}$

$\Psi_{\beta \gamma}=\lambda_{\gamma}^{2} \alpha_{1}+\left(\lambda_{\gamma}^{2} \lambda_{\beta}^{2}+\lambda_{\gamma}^{4}\right) \alpha_{2}+\alpha_{3}+\left(\lambda_{\beta}^{2}+\lambda_{\gamma}^{2}\right) \alpha_{4}+\left(\lambda_{\beta}^{4}+\lambda_{\gamma}^{4}\right) \alpha_{5} \quad(\beta \neq \gamma)$.

$\alpha_{1}$ and $\alpha_{2}$ are real functions of the strain invariants $I_{1}, I_{2}, I_{3}$ for the pure homogeneous deformation (2.1), defined by

$$
I_{1}=\lambda_{1}^{2}+\lambda_{2}^{2}+\lambda_{3}^{2}, \quad I_{2}=\lambda_{2}^{2} \lambda_{3}^{2}+\lambda_{3}^{2} \lambda_{1}^{2}+\lambda_{1}^{2} \lambda_{2}^{2}, \quad I_{8}=\lambda_{1}^{2} \lambda_{2}^{2} \lambda_{3}^{2},
$$

and $\alpha_{3}, \alpha_{4}, \alpha_{5}$ are real functions of these invariants and of $i \omega$. The relation of the $\alpha$ 's to the constants occurring in the Rivlin-Ericksen constitutive equation are discussed in [1].

1 In the previous paper [1], the quantities which we denote $\alpha_{1}, \alpha_{2}, \cdots, \alpha_{5}$ are denoted by $\tilde{\alpha}_{1}, \tilde{\alpha}_{2}, \cdots, \tilde{\alpha}_{5}$ and those which we denote $I_{1}, I_{2}, I_{3}$ are denoted by $I_{1}, I_{2}, I_{3}$. 
3. A particular class of waves. For the wave described by (2.2) and 2.3), the planes of constant phase are ${ }^{2}$

$$
S_{1}^{+} X_{1}+S_{2}^{+} X_{2}=\text { constant }
$$

and the planes of constant amplitude are

$$
S_{1}^{-} X_{1}+S_{2}^{-} X_{2}=\text { constant. }
$$

These are orthogonal if

$$
S_{1}^{+} S_{1}^{-}+S_{2}^{+} S_{2}^{-}=0,
$$

i.e. if

$$
S_{2}^{-} / S_{1}^{+}=-S_{1}^{-} / S_{2}^{+}=\lambda
$$

where $\lambda$ is real. We consider here the particular case when $\lambda=1$. Then

$$
S_{2}=i S_{1}
$$

and, introducing the notation $S_{1}=h_{3},(2.3)$ becomes

$$
u_{i}=U \delta_{i 3} \exp \left[i \omega h_{3}\left(X_{1}+i X_{2}\right)-t\right] .
$$

Introducing (3.5) into (2.4), we obtain

$$
h_{3}^{2}\left(\Psi_{31}-\Psi_{32}\right)=\rho .
$$

For waves of analogous types polarized in the $x_{1}$ and $x_{2}$ directions, the displacements $\epsilon u_{i}$ are given respectively by

$$
u_{i}=\epsilon \delta_{i 1} \exp \left[i \omega h_{1}\left(X_{2}+i X_{3}\right)-t\right]
$$

and

$$
u_{i}=\epsilon \delta_{i 2} \exp \left[i \omega h_{2}\left(X_{3}+i X_{1}\right)-t\right],
$$

where $h_{1}$ and $h_{2}$ satisfy the equations

$$
h_{1}^{2}\left(\Psi_{12}-\Psi_{13}\right)=\rho
$$

and

$$
h_{2}^{2}\left(\Psi_{23}-\Psi_{21}\right)=\rho .
$$

From (2.5), we see [1] that

$$
\Psi_{31}+\Psi_{12}+\Psi_{23}=\Psi_{13}+\Psi_{21}+\Psi_{82} .
$$

From (3.7) and (3.9), we obtain, using (3.10), the universal relation

$$
\left(1 / h_{1}^{2}\right)+\left(1 / h_{2}^{2}\right)+\left(1 / h_{3}^{2}\right)=0 .
$$

In a previous paper [1] the propagation of principal transverse waves has been discussed. We denote by $S_{\alpha \beta}$ the complex slowness vector for a wave polarized in the direction of the $x_{\alpha}$-axis, for which the planes of constant phase and planes of constant amplitude are both normal to the $x_{\beta}$-axis $(\alpha \neq \beta)$. It was shown in [1] that

$$
S_{\alpha \beta}^{2}=\rho / \Psi_{\alpha \beta} \quad(\alpha \neq \beta) .
$$

2 The superscripts + and - are used to denote the real and imaginary parts of a complex quantity. 
From (3.7) and (3.12), we obtain

$$
\left(1 / h_{3}^{2}\right)=\left(1 / S_{31}^{2}\right)-\left(1 / S_{32}^{2}\right) .
$$

Analogously we have the two further relations

$$
\left(1 / h_{2}^{2}\right)=\left(1 / S_{23}^{2}\right)-\left(1 / S_{21}^{2}\right), \quad\left(1 / h_{1}^{2}\right)=\left(1 / S_{12}^{2}\right)-\left(1 / S_{13}^{2}\right) .
$$

4. Elastic material. If the material considered is elastic, then [1]

$$
\alpha_{3}=\alpha_{4}=\alpha_{5}=0
$$

and $\alpha_{1}$ and $\alpha_{2}$ are derivable from a strain-energy function, $\Sigma$, per unit mass. The latter is a function of $I_{1}, I_{2}$ and $I_{3}$, and $\alpha_{1}$ and $\alpha_{2}$ are related to it by the formulae

$$
\begin{aligned}
& \alpha_{1}=2 \rho\left(\left(\partial \Sigma / \partial I_{1}\right)+I_{1}\left(\partial \Sigma / \partial I_{2}\right)\right), \\
& \alpha_{2}=-2 \rho\left(\partial \Sigma / \partial I_{2}\right) .
\end{aligned}
$$

Using (4.1), Eq. (2.5) becomes

$$
\Psi_{\beta \gamma}=\lambda_{\gamma}^{2} \alpha_{1}+\left(\lambda_{\gamma}^{2} \lambda_{\beta}^{2}+\lambda_{\gamma}^{4}\right) \alpha_{2} \quad(\beta \neq \gamma) .
$$

Then, with (4.2),

$$
\Psi_{31}-\Psi_{32}=\left(\lambda_{1}^{2}-\lambda_{2}^{2}\right)\left(\alpha_{1}+I_{1} \alpha_{2}\right)=2 \rho\left(\lambda_{1}^{2}-\lambda_{2}^{2}\right)\left(\partial \Sigma / \partial I_{1}\right) .
$$

With (3.7), we obtain

$$
1 / h_{3}^{2}=2\left(\lambda_{1}^{2}-\lambda_{2}^{2}\right)\left(\partial \Sigma / \partial I_{1}\right) .
$$

Analogous results can be obtained for $1 / h_{2}^{2}$ and $1 / h_{1}^{2}$ and they enable us, in principle, to determine $\partial \Sigma / \partial I_{1}$ for specified values of $\lambda_{1}, \lambda_{2}$ and $\lambda_{3}$ and, hence, for specified values of $I_{1}, I_{2}, I_{3}$ from measurements of $h_{1}, h_{2}$, or $h_{3}$ for waves of the type considered. We note, from (4.5) and the two analogous equations, that

$$
h_{1}^{2}\left(\lambda_{2}^{2}-\lambda_{3}^{2}\right)=h_{2}^{2}\left(\lambda_{3}^{2}-\lambda_{1}^{2}\right)=h_{3}^{2}\left(\lambda_{1}^{2}-\lambda_{2}^{2}\right) .
$$

It is not, in fact, necessary to make assumptions on the material as stringent as those implied by ideally elastic behavior in order to arrive at Eq. (4.6). From (2.5) we have

$$
\Psi_{31}-\Psi_{32}=\left(\lambda_{1}^{2}-\lambda_{2}^{2}\right)\left[\alpha_{1}+I_{1} \alpha_{2}+\alpha_{4}+\left(\lambda_{1}^{2}+\lambda_{2}^{2}\right) \alpha_{5}\right] .
$$

We see that if $\alpha_{5}=0$,

$$
\frac{\Psi_{31}-\Psi_{32}}{\lambda_{1}^{2}-\lambda_{2}^{2}}=\alpha_{1}+I_{1} \alpha_{2}+\alpha_{4}
$$

and from this and two analogous relations, we obtain, with (3.7) and (3.9), the relations (4.6). However, from a physical standpoint, the imposition of the restriction $\alpha_{5}=0$ seems somewhat arbitrary.

5. Energy flux. The mean energy flux vector $R_{B}$ is defined in the following manner. $R_{1}$ is the rate, measured per unit area, at which energy crosses an element of area normal to the $x_{1}$-axis. Analogous meanings attach to $R_{2}$ and $\Omega_{3}$. With this definition it has been shown (see Eq. (6.11) of [3]) ${ }^{3}$ that for the wave described by (2.3),

$$
R_{B}=\frac{1}{2} \epsilon^{2} \omega^{2} \exp \left[-2 \omega\left(S_{1}^{-} X_{1}+S_{2}^{-} X_{2}\right)\right] U^{2}\left[\left(\Psi_{31} S_{1}\right)^{+} \delta_{B 1}+\left(\Psi_{32} S_{2}\right)^{+} \delta_{B 2}\right] \text {. }
$$

3 To bring the notation in Eq. (6.11) of [3] into conformity with that of the present paper, replace ' $R_{B}$ by $R_{B}, i S_{A}^{\prime}$ by $S_{A}, X_{A}^{\prime}$ by $X_{A}$ and $A$ by $U$. 
We note that this vector is parallel to the vector with components $\left[\left(\Psi_{31} S_{1}\right)^{+},\left(\Psi_{32} S_{2}\right)^{+}, 0\right]$.

For the wave described by (3.6), we take $\left(S_{1}, S_{2}\right)=\left(h_{3}, i h_{3}\right)$. The vector $R_{B}$ is then parallel to $\left\{\left(\Psi_{31} h_{3}\right)^{+},-\left(\Psi_{32} h_{3}\right)^{-}, 0\right\}$.

We note that for the wave described by (3.6), the planes of constant phase are normal to the vector $\left(h_{3}^{+},-h_{3}^{-}, 0\right)$ while the planes of constant amplitude are normal to the vector $\left(h_{3}^{-}, h_{3}^{+}, 0\right)$.

In the particular case when $h_{3}^{-}=0$, i.e. $h_{3}$ is real, the normals to the planes of constant phase and constant amplitude are along the $x_{1}$ and $x_{2}$ directions respectively, while the mean energy flux vector is parallel to $\left(\Psi_{31}^{+}, \Psi_{32}^{-}, 0\right)$. Thus, even in this case the ray direction is not, in general, in the direction of the normal to the planes of constant phase, nor in the direction of the normal to the planes of constant amplitude. It is seen that, in this case, the expression (5.2) for $R_{B}$ becomes

$$
Q_{B}=\frac{1}{2} \epsilon^{2} \omega^{2} \exp \left(-2 \omega h_{3} X_{2}\right)\left(\Psi_{31}^{+} \delta_{B 1}-\Psi_{32}^{-} \delta_{B 2}\right) h_{3} \cdot
$$

[1] M. A. Hayes and R. S. Rivlin, J. Acoust. Soc. Amer. 46, 610 (1969)

[2] F. J. Lockett, J. Mech. Phys. Solids 10, 53 (1962)

[3] M. A. Hayes and R. S. Rivlin (pending publication) 